\title{
4. \\ Anwendungen der Statik auf die Lehre von den geometrischen Verwandtschaften.
}

(Vom Hro. Prof. A. F. Möbius zn Leipzig.)

Unter den Aufgaben der elementaren Statik ist die wichtigste unstreitig diejenige, welche die Bedingungen des Gleichgewichts zwischen Kräften verlangt, die nach gegebenen Richtungen auf gegebene Puncte eines freibeweglichen festen Körpers wirken : zwischen Kräften also, deren Angriffspuncte in unveränderlichen Entfernungen von einander stehen. Diese Bedingung für die Angriffspuncte läfst sich auch dadurch ausdrücken, dafs sie eine sich immer gleich und ähnlich bleibende Figur bilden sollen; und man kann hierdurch veranlafst werden, nach den Bedingungen des Gleichgewichts zu fragen, wenn die Angriffspuncte nur dergestalt mit einander verbunden sind, dafs sie auch in jede andere der anfänglichen blofs ähnliche Figur gebracht werden können.

Die hierdurch sich bildende Aufgabe habe ich für den Fall, wenn die Puncte und die auf sie wirkenden Kräfte in einer Ebene enthalten sind, bereits in meinem "Lehrbuche der Statik" zu lösen gesucht. Ich dachte mir nänlich ein System von Geraden, welche sich unter unveränderlichen Winkeln in einem beweglichen Puncte treffen, mit einem andern Systeme von derselben Beschaffenheit dergestalt verbunden, dafs eine Gerade des eineu Systems mit einer Geraden des anderen zusammenfiel und längs derselben verschiebbar war, und suchte nun die Bedingungen des Gleichgewichts zwischen Kräften, welche ich auf die gegenseitigen Durchschnitte der Geraden des einen und andern Systems wirken liefs; denn offenbar mufsten diese Puncte bei der angenommenen Beweglichkeit eine sich ähnlich bleibende Figur bilden.

Es giebt aber, wie ich in meinem "Barycentr. Calcul" gezeigt habe, aufser der Gleichheit und Aehnlichkeit und der blofsen Aehnlichkeit noch einige andere Verwandtschaften, in denen Figuren zu einander stehen kōnnen, und welche gleichfalls in das Gebiet der niedern Geometrie gehören; namentlich die blofse Gleichbeit, die Affinität und die Verwandtschaft der Collineation. 
Man kann daher auf analoge, Weise die Bedingungen des Gleichgewichts zu erforschen suchen, wenn die Beweglichkeit der Angriffspuncte der Kräfte dadurch hestimmt wird, dafs sie eine sich immer blofs gleich, oder affin, oder collinear verwandt bleibende Figur bilden sollen. Da je zwei einander gleiche und 'ähnliche Figuren auch in jeder entferntern Verwandtschaft zu einander stehen, so werden die bekannten Bedingungen des Gleichgewichts, welche bei Unveränderlichkeit der gegenseitigen Entfernungen der Angriffspuncte Statt.finden, auch bei jeder entferntern Verwandtschaft, an welche die Beweglichkeit der Angriffspuncte gebunden wird, wiederkehren, zu ilanen aber nẹue, von der Natur der jedesmaligen Verwandtschaft abhängige, hinzutreten, und dieses in desto gröfserer Zahl, je entfernter die Verwandtschaft, und je gröfser folglich die Beweglichkeit der Puncte ist.

Die im Obigen gedachte Untersuchung in Betreff sich ähnlich bleibender ebener Figuren habe ich daher späterhin noch auf die Aehnlichkeit im Raume und auf die entfernteren Verwandtschaften ausgedehnt, und dieses vorzüglich mit aus dem Grunde, weil zu erwarten stand, auf diesem Wege zu einigen neuen Eigenschaften der Verwandtschaften selbst zu gelangen. Ich veröffentliche jetżt diese Untersuchungen in der Hoffnung, dals es vielleicht auch Andern angenehm sein dürfte, die Gleichgewichtsbedingangen, welche bei den entferntern Verwandtschaften hinzutreten, und die etwaigen daraus gezogenen geometrischen Folgerungen kennen zu lernen. Uebrigens habe ich mich hier stets des Princips der virtuellen Geschwindigkeiten, als des einfachsten dabei anzuwendenden Mittels, bedient und mit Hülfe desselben die früherę Untersuchung sich ähnlich bleibẹndẹr Figuren von Neuem angestellt.

\section{Bedingungen des Gleichgewichts bei sich ähnlich bleibenden Figuren.}

1. In Bezug auf zwei rechtwinklige Goordinatensysteme in einer Ebene seien $x, y$ und $t, u$ die Coordinaten eines Punctes der Ebene. Man hat alsdann :

$$
\begin{aligned}
& x=r+t \cos \alpha-u \sin \alpha \\
& y=g+t \sin \alpha+u \cos \alpha
\end{aligned}
$$

wo $f$ und $g$ die Coordinaten des Anfangspunctes des Systems der $t$ und $u$, Crelle's Journal d. M. Bd. XXI. Hit. 1. 
in Bezug auf das System der $x$ und $y$ sind, $\alpha$ aber der Winkel "der Axe der $t$ mit der Axe der $x$ ist.

Setzen wir nun, dafs auf gleiche Weise noch mehrere andere Puncle der Ebene auf beide Coordinatensysteme bezogen seien, dafs diese Puncte gegen das System der Axen $t$ und $u$ eine unverăiderliche Lage háben, dafs aber dieses Axensystem sammt den Puncten seiner Lage gegen das in der Ebene rubig bleibende System der Axen $x$ und $y$ beliebig ändern könne: so sind in den obigen Gleichungen $t$ und $u$ constant, dagegen $f, g, \alpha$ beliebig veränderlich, und damit auch $x$ und $y$ veränderlich.

Wir wollen jetzt die Lage der Puncte gegen die Axen der $t$ und $u$ nicht mehr constant, jedoch nur dergestalt veränderlich ainnehmen, dafs die von ihnen mit den Axen gebildete Figur sich immer ähnlich bleibt. Zu dem Ende haben wir nur für $t$ und $u \ldots . \frac{t}{n}$ und $\frac{u}{n}$ zu schreiben, wo $n$, eben so wie $f, g$ und $\alpha$, von einem Puncte zum andern gleich grofs, aber mit der Zeit beliebig veränderlich ist. Hiermit werden die obigen Gleichungen, wenn wir noch $a$ und $b$ für $n f$ und $n g$ setzen :

$$
\text { 1. }\left\{\begin{array}{l}
n x=a+t \cos \alpha-u \sin \alpha, \\
n y=b+t \sin \alpha+u \cos \alpha .
\end{array}\right.
$$

Durch diese Gleichungen, in denen nur $t$ und $u$ constant sind, werden daher Puncte $(x, y)$ in der Ebene bestinmt, die ihre Lage dergestalt anf jede Weise ändern können, dals die von ihnen gebildete Figur sich immer ähnlich bleibt.

Die Differentiation dieser Gleichungen giebt :

$$
\text { 2. }\left\{\begin{array}{l}
n d x+x d n=d a-(n y-b) d a, \\
n d y+y d n=d b+(n x-a) d \alpha .
\end{array}\right.
$$

Wirkt nun auf jeden Punct $(x, y)$ des Systems eine Kraft $(\boldsymbol{X}, \boldsymbol{Y})$, d. h. eine Kraft, welche, nach den Axen der $x, y$ zerlegt, die Kräfte $\boldsymbol{X}$ und $\boldsymbol{Y}$ giebt, so hat man nach dem Princip der virtuellen Geschwindigkeiten als Bedingung des Gleichgewichts zwischen allen diesen Kräften die Gleichung $\Sigma(X d x+Y d y)=0$, und wenn man darin für $d x$ und $d y$ ihre Werthe aus (2) substituirt:

$$
\begin{gathered}
(d a+b d a) \Sigma X+(d b-a d \alpha) \Sigma \boldsymbol{Y}+n d \alpha \Sigma(\boldsymbol{Y} x-\boldsymbol{X} \boldsymbol{y}) \\
-d n \Sigma(X x+\boldsymbol{Y} y)=0 .
\end{gathered}
$$

Da aber die Differentiale $d a, d b, d \alpha$ und $d n$ von einander ganz unab- 
4. Möbius, Anwendungen der Statik auf geometrische Verwandtschaften.

hängig sind, so zerfällt diese Gleichung in folgende vier einzelne :

$$
\boldsymbol{\Sigma} \boldsymbol{X}=0, \quad \Sigma \boldsymbol{Y}=0, \quad \boldsymbol{\Sigma}(\boldsymbol{Y} \boldsymbol{x}-\boldsymbol{X} \boldsymbol{y})=0, \quad \boldsymbol{\Sigma}(\boldsymbol{X} \boldsymbol{x}+\boldsymbol{Y} \boldsymbol{y})=0 \text {. }
$$

Die drei ersten derselben sind die bekannten Bedingungen des Gleichgewichts, wenn die gegenseitige Lage der Angriffspuncte der Kräfte unveränderlich ist. Die vierte kommt wegen der gemachten Voraussetzung hinzu, dafs die gegenseitige Lage der Puncte zwar veränderlich sein soll, jedoch nư so, dafs die von ihnen gebildete Figur sich immer ähnlich bleibt.

2. Zusatz. Nach dem 5. 122. meines Lehrbuchs der Statik ergeben sich diese vier Gleichungen als Bedingungen des Gleichgewichts zwischen Kräften, welche auf Puncte in einer Ebene wirken, auch in dem Falle, wenn die gegenseitige Lage der Puncte unveränderlich ist und wenn das Gleichgewicht nicht blofs bei einer bestimmten Lage des Systems der Puncte in der Ebene Statt findet, sondern auch noch bei jeder beliebigen Drehung in der Ebene, während die Kräfte mit parallel bleibenden Richtungen 'und unveränderten Intensitäten auf dieselben Puncte zu wirken fortfahren, noch besteht. Hiernach kann man folgende zwei Sätze (ebend. \$5. '235. u. 236.) aufstellen :

, Sind mehrere Puncte in einer Ebene dergestalt beweglich, da/s die von ihnen gebildete Figur sich immer ähnlich bleibt, und halten sich Kräfte, welthe auf sie in der Ebene wirken, das Gleichgewicht, so herrscht auch noch Gleichgewicht bei jeder andern Lage, welche man den Puncten zufolge iltrer Beweglichkeit geben kann, wenn nur die Kräfte ihren anfänglichen Richtungen parallel bleiben;" und umgekehrt:

, Sind Krïfle, welche auf fest mit einander verbundene Puncte in einer Ebène wirken, im Gleichgewichte, und dauert dasselbe noch fort, wenn das System' der Puncte in seiner Ebene beliebig verschoben wird, die Kräfte aber parallel mit ihren anfünglichen Richtungen fortwirken, so wird das Gleichgewicht auch nicht unterbrochen, wenn man den Puncten eine solche gegènseitige Beweglichkeit noch beilegt, bei welcher die von ihnen gebildete Figur sich immer ähnlich bleibt.",

3. Un von dieser Theorie eine Anwendung auf die einfachsten Falle zu machen, wollen wir zuerst setzen, das System bestehe nur aus zwivei Puncten $(x, y)$ und $\left(x_{1}, y_{1}\right)$, auf welche resp. die Kräfte $(X, Y)$ und $\left(\boldsymbol{X}_{1}, \boldsymbol{Y}_{1}\right)$ wirken. Die vier Bedingungen des Gleichgewichts sind alsdann:

$$
\begin{gathered}
\boldsymbol{X}+\boldsymbol{X}_{1}=0, \quad \boldsymbol{Y}=\boldsymbol{Y}_{1}=0, \quad \boldsymbol{Y} x-\boldsymbol{X}_{y}+\boldsymbol{Y}_{1} x_{1}-\boldsymbol{X}_{1} y_{1}=0 \\
\boldsymbol{X}_{x}+\boldsymbol{Y} \boldsymbol{y}+\boldsymbol{X}_{1} x_{1}+\boldsymbol{Y}_{1} y_{1}=0 .
\end{gathered}
$$


Die Elimination von $\boldsymbol{X}_{1}$ und $\boldsymbol{Y}_{1}$, aus dièsien Gleichungen giebt :

$$
\boldsymbol{Y}\left(x-x_{1}\right)-\boldsymbol{X}\left(y-y_{1}\right)=0, \ldots X\left(x-x_{1}\right)+\boldsymbol{Y}\left(y-y_{1}\right)=0 ;
$$

und wenn wir hieraus noch $X$ und $\boldsymbol{Y}$ wegschaffen:

$$
\left(x-x_{1}\right)^{2}+\left(y-y_{1}\right)^{2}=0
$$

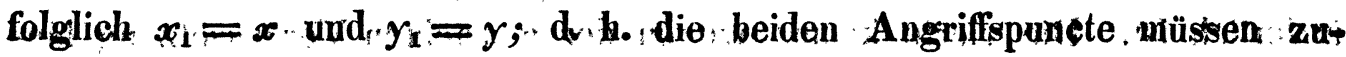
sammenfallen:" Auch folgt dieses schon aus der Natur der Sache selbst. Denn ein System von zwęi nicht zusammenfallepden; Puncten buleibt bei jeder Aenderung ihrer gegenseitigen Lage sich ähnlich. .: Zwoi Kräfte aber, angebracht an zwei Puncten, deren gegenseitige Lage beliebig veränderlich ist, können nicht im Gleichgewichte sein.

Anders verhält es sich wenn zu den zwei Puncten ein dritter $\left(x_{2}, y_{2}\right)$, getrieben von der Kraft: $\left(\boldsymbol{X}_{2} ; \boldsymbol{Y}_{2}\right)$, hinzukommat. Die vier Gleichgewichtsbedingungen sind in diesem Falle :

$$
\text { (A.) }\left\{\begin{array}{l}
X+X_{1}+X_{2}=0, Y Y_{1}+Y_{2}=0 \\
Y x-X y+Y_{1} x_{1}-X_{1} y_{1}+Y_{2} x_{2}-X_{2} y_{2}=0 \\
X x+Y y+X_{1} x_{1}+Y_{1} y_{1}+X_{2} x_{2}+Y_{2} y_{2}=0
\end{array}\right.
$$

Man multiplicire von diesen Gleichungen die erste, zweite..und vierte resp. mit $-f,-g$ und 1 , addire sie hierauf und setze zur Bestimmung von $f$ und $g$ :

$$
\text { (a.) } \quad X(x-f)+Y(y-g)=0, \quad X_{1}\left(x_{1}-f\right)+Y_{1}\left(y_{1}-g\right)=0
$$

so ist auch

$$
\text { (b.) } \quad X_{2}\left(x_{2}-f\right)+Y_{2}\left(y_{2}-g\right)=0 \text {. }
$$

Betrachtet man nun $f, g$ als die Coordinaten eines Punctes und bezeichnet die Puncte $(x, y),\left(x_{1}, y_{1}\right),\left(x_{2}, y_{2}\right),(f, g)$ mit $\boldsymbol{A}, \boldsymbol{A}_{1}, \boldsymbol{A}_{2}, \boldsymbol{F}$ und die drei Kräfte $(X, Y)$ etc. mit $P, P_{1}, P_{2}$, so sind nach (a.) die Linien $\boldsymbol{F A}$ und $F A_{1}$ resp. auf $\boldsymbol{P}$ und $\boldsymbol{P}_{1}$ rechtwinklig, d. h. $\boldsymbol{F}$. ist der Durchschuitt der auf $\boldsymbol{P}$ und $\boldsymbol{P}_{1}$ in $\boldsymbol{A}$ und $\boldsymbol{A}_{1}$ errichteten Perpendikel. Den so bestimmten Pųnct $F$ mufs aber nach $(b$.$) auch das auf P_{2}$ in $A_{2}$ errichtete Perpendikel treffen, und die Bedingung wegen der danernden Aehnlichkeit besteht hiernach darin, dafs sich die drei auf den Kräften in ihren Augriffspuncten errichteten Perpendikel in einem Puncte $\boldsymbol{F}$ schneiden.;

Noch anders kann diese Bedingung ausgedrückt werden, weun man sich erimert, dafs die Richtungen dreier sich das Gleichgewicht haltenden Kräfte sich in einem Puncte begegnen (eine Eigenschaft, welche auch unmittelbar aus; den drei ersten der obigen vier Gleichungen ( $\boldsymbol{A}_{\text {. }}$ ) hergeleitet werden kann). Ist nun $\boldsymbol{K}$, dieser gemeinschaftliche punct der 
Richtungen von $\boldsymbol{P}, \boldsymbol{P}_{1}, \boldsymbol{P}_{2}$, so sind dem Vorigen zufolge $\boldsymbol{F A K}, \boldsymbol{F A} \boldsymbol{A}_{1} \boldsymbol{K}$, $F A_{2} K$ rechte Winkel, und $K$ liegt folglich mit $A_{1} A_{1}, A_{2}$ in einem $K$ reise.

„Sollen demnach in einer Bbene drei Kräfte an drei Puncten, welche ein sich ähnlich bleibendes Dreieck zu bilden genöthigt sind, in Gleichgewichte sein, so mufs; nächst den Bedingungen des Gleichgewichts für den Fall, wenn die Puncte in unabänderliclier Entfernung von einander sind, auch noch die erfüllt werden, dafs die drei Puncte mit demjenigen, in welchem sioh die Riohtungen der drei Kräfte schneiden, in einem Kreise liegen."

Eine leichte Folgerung hieraus ist, dafs die Winkel, welche die Kräfte mit einanđer bilden, den Supplementen der Winkel des Dreiecks $\boldsymbol{A}_{\boldsymbol{A}_{1}} \boldsymbol{A}_{2}$ gleich sind, nämlich der Winkel der Kräfte an $\boldsymbol{A}_{1}$ und $\boldsymbol{A}_{2}$, = $180^{\circ}-\boldsymbol{A}_{1} \boldsymbol{A} \boldsymbol{A}_{2}$, etc. und daf's deshalb, und weil beim Gleichgewichte zwischen drei Kräften jede Kraft dem Sinus des von den beiden andern Kräften gebildeten Winkels proportional ist, die Kräfte sich wie die ihren Angriffspuncten $\boldsymbol{A}, \boldsymbol{A}_{1}, \boldsymbol{A}_{2}$ gegenüber liegenden Seiten des Dreiecks $\boldsymbol{A A}_{1} \boldsymbol{A}_{2}$ verhalten.

Man bemerke hierbei noch, wie von dem Unslande, dafs der gegenseitige Durchschnitt der drei Kräfte mit ihren Angriffspuncten in einem Kreise liegt, die Fortdauer des Gleichgewichts bei der Drehung des Dreiecks $\boldsymbol{A} A_{1} A_{2}$ in seiner Ebene eine unmiltelbare Folge ist. Ob nämlich das Dreieck gedreht wird, während jede Kraft ihrer anfänglichen Richtung parallel bleibt, oder ob das Dreieck in Ruhe bleibt und jede Kraft un einerr gleich grofsen Winkel um ihren Angriffspunct gedreht wird, kommt hier, wo es sich nur um die gegenseitige Lage handelt, offenbar auf dasselbe hinaus. Wenn aber drei von $\boldsymbol{A}, \boldsymbol{A}_{1}, \boldsymbol{A}_{2}$ ausgehende Geraden um diese Puncte um gleich grofse Winkel gedreht werden, so rücken ihre Durchschnitte mit dem durch $\boldsymbol{A}, \boldsymbol{A}_{1}, \boldsymbol{A}_{2}$ zu beschreibenden Kreise um gleicli grofse Bogen fort. Wenn folglich diese drei Geraden sich anfangs in einem Puncte $\boldsymbol{K}$ des Kreises schnitten, so wird dieses auch nach der Drehung noch der Fall sein; folglich u. s. w.

4. Die im Vorigen für eine Ebene angestellien Untersuchungen wollen wir jetzt auf den Raum ausdehneu. Seien daher bei einem System von Puncten im Raume die Coordinaten eines derselben in Bezug auf zwei rechtwinklige Axensysteme $x, y, z$ und $t, u, v$, so kann man setzen: 
4. Möbius., Anwendungen der Statik auf geometrische Vernatdtschaften.

$$
\begin{aligned}
& x=f+\alpha t+\alpha^{\prime} u+\alpha^{\prime \prime} v, \\
& y=g+\beta t+\beta^{\prime} u+\beta^{\prime \prime} v, \\
& z=h+\gamma t+\gamma^{\prime} u+\gamma^{\prime \prime} v,
\end{aligned}
$$

wo $a=\cos x^{\wedge} t, \alpha^{\prime}=\cos x^{\wedge} u$, etc. und $f, g, h$, die Coordinaten des Anfangspunctes des Systems der $t, u, v$ in Bezug auf das System der $x, y, z$ sind.

Hieraus lärst sich, wie im Obigen, weiter folgern, dafs, wenn man 1. $\quad n x=a+\alpha t+\alpha^{\prime} u+\alpha^{\prime \prime} v, \quad n y=b+\beta t+\ldots ., n z=c+\ldots$. selzt und dabei $t, u, v$ constant, $n, a, b, c$ aber und $\alpha, \beta^{\prime}, \gamma^{\prime \prime}$, wovon die übrigen $\alpha^{\prime}, \alpha^{\prime \prime}, \beta$, etc. auf bekannte Weise abhängen, veränderlich annimmit: dafs dann das System der Puncte, zu welchen $(x, y, z)$ gehört, bei beliebiger Aenderung von $n, a, b, c, \alpha, \beta^{\prime \prime}, \gamma^{\prime \prime}$ seine Lage sowohl als Gröfse beliebig ändert, sich dabei aber stets ähnlich bleibt.

Ist nun $(\boldsymbol{X}, \boldsymbol{Y}, \boldsymbol{Z})$ die auf den Punct $(\boldsymbol{x}, \boldsymbol{y}, \boldsymbol{z})$ wirkende $\mathrm{Kraft}$, und soll zwischen ihr und den an den übrigen Puncten des Systems angebrachten Kräften Gleichgewicht herrschen, so mufs bei allen Verrückungen, deren das System fähig ist,

$$
\Sigma(\boldsymbol{X} d \boldsymbol{x}+\boldsymbol{Y} d \boldsymbol{y}+\boldsymbol{Z} d \boldsymbol{d})=0
$$

sein. Es findet sich aber, wenn man hierin für $d x, d y, d z$ ihre aus der Differentiation von (1) fliefsenden Werthe setzt:

$$
\text { 2. } \begin{gathered}
\Sigma(\boldsymbol{X} x+\boldsymbol{Y} y+\boldsymbol{Z} z) d n-\Sigma \boldsymbol{X}\left(\boldsymbol{d} a+t d \alpha+u d \alpha^{\prime}+\ldots . .\right) \\
-\Sigma \boldsymbol{Y}(\boldsymbol{d} \boldsymbol{b}+t d \beta+\ldots .)-\Sigma \boldsymbol{Z}(\boldsymbol{d} \boldsymbol{c}+\ldots . .)=0 .
\end{gathered}
$$

Da das Differential $d \boldsymbol{n}$ von den übrigen hierin vorkommendeu Differentialen unabhängig ist, so ergiebt șich, als erste Bedingung des Gleichgewichts,

$$
\Sigma\left(\boldsymbol{X} \boldsymbol{x}+\boldsymbol{Y} \boldsymbol{y}+\boldsymbol{Z}_{z}\right)=0 .
$$

Um die übrigen Bedingungsgleichungen zu erhalten, hat man in dem übrigen Theile der Gleichung (2) die Coordinaten $t, u, v$ mittelst (1) durch $x, y, z$ auszudrücken und dann noch die 9 Differentiale $d \alpha, d \alpha^{\prime}, \ldots d \gamma^{\prime \prime}$ auf drei von einander unabhängige zu reduciren. Ohne aber diese etwas weilläufige Rechnung anzustellen, sieht man schon im Voraus, dafs die auf solche Weise zu erhaltenden Bedingungsgleichungen keine andern als die bekannten sechs sein können, welche Statt finden müssen, wenn die gegenseitige Lage der Angriffspuncte unveränderlich ist. Denn da der übrige Theil der Gleichung (2) von $n$ unabhängig ist, so müssen die aus ihm zu folgernden Gleichungen einerlei sein mit denen, welche man erhält, wenn man $n$ constant setzt. Ist aber $n$ constant, so bleibt sich das System der Puncte $(x, y, z)$ nicht blofs ähnlich, sondern auch gleich; folglich u. s. w. 
4. Möbius, Anwendungen der Statik auf geometrische Verwandtschaften.

Der Bedingungsgleichungen für das Gleichgewicht an einem sich ähnlich bleibenden Systeme von Puncten im Raume giebt es demnach in Allem sieben: nämlich die bekannten sechs

$$
\begin{aligned}
& \Sigma X=0, \\
& \Sigma Y=0 \text {, } \\
& \Sigma Z=0, \\
& \Sigma(\boldsymbol{Y} z-\boldsymbol{Z} \boldsymbol{y})=0, \\
& \Sigma\left(Z x-X_{z}\right)=0 \text {, } \\
& \Sigma\left(X \boldsymbol{y}-Y_{\boldsymbol{x}}\right)=0 \text {, }
\end{aligned}
$$

und die vorhin zuerst gefundene:

$$
\Sigma(X x+Y y+Z z)=0 .
$$

5. Zusätze. a) Bezeichnet $\boldsymbol{A}$ den Punct $(x, y, z)$ des Systems, $\boldsymbol{P}$ die auf ihn wirkende $\mathbf{K r a f t}(X, Y, Z)$, und $\boldsymbol{O}$ den Anfangspunct der Coordinaten, so ist der summatorische Ausdruek

$$
\Sigma(\boldsymbol{X} \boldsymbol{x}+\boldsymbol{Y} \boldsymbol{y}+Z \boldsymbol{z})=\Sigma \boldsymbol{O A} \cdot \boldsymbol{P} \cos \boldsymbol{O} \boldsymbol{A}^{\wedge} \boldsymbol{P}
$$

er ist folglich unabhängig von dem durch $O$ gelegten Systeme der Coordinatenaxen, was auch für Kräfte $\boldsymbol{P}$ auf die Puncte $\boldsymbol{A}$ wirken mögen. Gegenwärtig aber, wo zugIeich $\Sigma X=0, \Sigma Y=0, \Sigma Z=0$ sein soll, ist jener Ausdruck auch von dem Anfangspuncte der Coordinaten unabhängig. Denn für einen neuen Anfangspunct, dessen Coordinaten in Bezug auf den alten, $=a, b, c$ sind, wird der Ausdruck

$$
=\Sigma(X(x-a)+Y(y-b)+Z(z-c)):
$$

und dieser ist von dem vorigen $\Sigma(X x+\ldots)$ um $a \Sigma X+b \Sigma y+c \Sigma Z$, das heifst um nichts verschieden.

Die specielle Bedingung, unter welcher Kräfte an einem Systeme von Puncten, welches sich immer ähnlich bleiben soll, im Gleichgewichte sind, kann hiernach folgendergestalt ausgedrückt werden :

,Wählt man beliebig einen Punct $(\boldsymbol{O})$ und multiplicirt jede Kraft $(\boldsymbol{P})$ in den $A b s t a n d ~ O P \cos O A^{\wedge} P$ ihres Angriffspunctes $(\boldsymbol{A})$ von einer durch den erstern Punct $(O)$ perpendiculär auf die Richtung der Kraft gelegten Ehene, so mufs die Summe dieser Producte Null sein."

b) Sind sämmtliche Kräfte mit einander parallel, und nimmt man mit ihnen die Axe der $z$ parallel an, so werden $X$ und $Y$ Null, und die obigen 7 Bedingungsgleichungen reduciren sich auf folgende vier:

$$
\Sigma Z=0, \quad \Sigma Z x=0, \quad \Sigma Z y=0, \quad \Sigma Z z=0 \text {; }
$$

d. h. der Angriffspunct jeder Kraft ist der Mittelpunct der jedesmal übrigen.

Denn sind aufser $Z$ die übrigen $K_{\text {räfte }} Z_{1}, Z_{2}, \ldots$ und $\left(x_{1}, y_{1}, z_{1}\right)$, $\left(x_{2}, y_{2}, z_{2}\right)$, etc. ihre Angriffspuncte, so kann man statt der letzten vier Gleichungen auch schreiben :

$$
\begin{array}{ll}
Z+\Sigma Z_{1}=0, & Z_{x}+\Sigma Z_{1} x_{1}=0, \quad Z y+\Sigma Z_{1} y_{1}=0 \\
& Z z+\Sigma Z_{1} z_{1}=0 .
\end{array}
$$


Hieraus folgt:

$$
x=\frac{\Sigma Z_{1} x_{1}}{\Sigma Z_{1}}, y=\frac{\Sigma Z_{1} y_{1}}{\Sigma Z_{1}}, z=\frac{\Sigma Z_{1} z_{1}}{\Sigma Z_{1}} .
$$

Die so bestimmten Werthe von $x, y, z$, sind aber die Coordinaten des Mittelpuncts der Kräfte $\boldsymbol{Z}_{1}, \boldsymbol{Z}_{2}, \ldots$

c) Liegen daher die Angriffspuncte aller parallelen Kräfte, bis auf einen, in einer Ebene, so mufs auch der letztere in dieser Ebene enthalten sein, wenn das System unter der Bedingung bleibender Aehnlichkeit in Gleichgewichte verharren soll. Denn der Mittelpunct eines Systems paralleler Kräfte, deren Angriffspuncte in einer Ebene liegen, ist gleichfalls in dieser Ebene begriffen.

Uebrigens sieht man von selbst, wie die jetzt gemachten Schlüsse mit gehöriger Modification auf den früher behandelten Fall anwendbar sind, wo die Puncte und die auf sie wirkenden Kräfte in einer und derselben Ebene enthalten waren.

6. Eine besondere Betrachtung wollen wir noch dem einfachen Falle widmen, wenn das System aus vier $\mathrm{Kräften} \boldsymbol{P}, \boldsymbol{P}_{1}, \boldsymbol{P}_{2}, \boldsymbol{P}_{3}$ bestelit, welche auf vier nicht in einer Ebene liegende Puncte $A, A_{1}, A_{2}, A_{3}$ wirken. Durch einen beliebigen fünften Punct $\boldsymbol{O}$ lege man vier Ebenen perpendicular auf die Richtungen 'von $P, P_{1}, P_{2}, P_{3}$ und nenne $D, D_{1}, D_{2}, D_{3}$ die Durchschnitte dieser Ebenen mit den Richtungen von $\boldsymbol{P}, \boldsymbol{P}_{1}, \ldots$ Alsdann ist nach No. 5. $a$. die specielle Bedingung des Gleichgewichts, welche bei der Annahme dauernder Aehnlichkeit des Systems der Angriffspuncte erfüllt werden mufs :

$$
D A . P+D_{1} A_{1} \cdot P_{1}+D_{2} A_{2} \cdot P_{2}+D_{3} A_{3} \cdot P_{3}=0 .
$$

Man wähle nun zum Puncte $O$ denjenigen, in welchem sich drei auf $P, P_{1}, P_{2}$ resp. in $\boldsymbol{A}, \boldsymbol{A}_{1}, \boldsymbol{A}_{2}$ perpendiculär gelegte Ebenen schneiden, so sind $D A, D_{1} A_{1}, D_{2} A_{2}$ einzeln Null. Zufolge der Bedingungsgleichung mufs daher bei dem also bestimmten $O$ auch $D_{3} A_{3}=0$ sein, d.h. die durch $O$ perpendicular auf $\boldsymbol{P}_{3}$ gesetzte Ebene mufs $\boldsymbol{P}_{3}$ in $\boldsymbol{A}_{3}$ treffen, und wir schliefsen hieraus :

„Sind vier Puncte in Raume dergestalt beweglich, dafs die von ihnen gebildete Figur sich immer ähnlich bleibt, und sollen vier auf sie wirkende Kräfte sich das Gleichgewicht halten, so mufs aufșer: den zun Gleichgewichte nöthigen Erfordernissen, wenn die Puncte fest mit einander verbunden sind, auch noch die Bedingung erfụllt werden, dafs die vier 
Ebenen, welche durch die vier Puncte, jede perpendicular auf der Richtung der den Punct treibenden Kraft, gelegt werden, sich in einem Puncte $(\boldsymbol{O})$ schneiden."

(1) Zum Gleichgewichte zwischen vier Kräften, deren Angriffspancte fest mit einander verbunden sind, ist unter anderen erforderlich, 'dals jede Gerade, welche die Richtungen dreier der vier Kräfte schneidet, auch der Richtung der vierten begegnet (Lehrb. der St. S. 99. a.). Wenn daher drei Richtungen, welche nicht in einer Ebene enthalten sind, sich in einem Puncte $K$ schneiden, so mufs auch die vierte Richtung den Punct $K$ treffen. Bei dieser speciellen Lage der Richtungen lärst sich die besondere Bedingung des Gleichgewichts, wegen der Aehnlichkeit, auf analoge Weise als wie oben bein Gleichgewichte zwischen drei Kräften, ausdrücken. Ess müssen nämlich die vier Winkel $O A K, O A_{1} K, O_{2} K, O A_{3} K$ rechte Winkel sein, d. h. „es mufs der gemeinschaftliche Durchschnitt der vier Richtungen in der durch die vier Angriffspuncte zu beschreibenden Kugelfläche liegen." 